\title{
Ball, Rafael: Wissenschaftskommunikation im Wandel. Von Gutenberg bis Open Science.
}

\author{
Wiesbaden: Springer VS 2021. 141 Seiten. Preis: $€ 34,99$ (e-book)
}

\section{Corinna Lüthje}

Angenommen: 6. März 2021 / Online publiziert: 15. März 2021

(C) Der/die Autor(en) 2021

Wir leben in einer denkwürdigen Zeit. Wissenschaft und ihre Kommunikation bekommen in der Pandemie eine Relevanz wie noch nie zuvor. Wissenschaftliche Begriffe und die Art und Weise, wie wissenschaftliches Wissen produziert, auf Qualität überprüft und verbreitet wird, sind zu einem Teil des Alltags geworden. In diese Zeit fällt die Publikation des Buches von Rafael Ball zu Wissenschaftskommunikation im Wandel.

$\mathrm{Zu}$ Beginn dieser Rezension muss angemerkt werden, dass der Begriff „Wissenschaftskommunikation“ im Titel ein wenig in die Irre führen kann - zumindest eine Leserschaft aus den Reihen der Kommunikations- und Medienwissenschaften und auch eine breitere Öffentlichkeit, die mit Wissenschaftskommunikation vor allem externe Kommunikation verbindet: Wissenschaftsjournalismus und Wissenschafts-PR. Auch im englischen Sprachraum werden diese Bereiche unter Science Communication gefasst. Ball behandelt in der Tradition der Informations- und Bibliothekswissenschaften das, was in den Bereich der Scholarly Communication fällt: wissenschaftliche Publikation bzw. formale Wissenschaftskommunikation. Ball definiert dies als ,den Vorgang der wissenschaftlichen Diskussionen und der Weiterverbreitung ihrer Inhalte“" (S. 24).

In diesem Buch wird die Entwicklung der wissenschaftsinternen Kommunikation von der Antike bis zur Gegenwart dargestellt. Man könnte fast von dem Versuch einer Kulturgeschichte der wissenschaftsinternen Kommunikation sprechen. Gesellschaftlicher Wandel und Medienwandel führten zu drei Paradigmenwechseln: von der mündlichen zur schriftlichen Kommunikation mit Aristoteles, von der briefbasierten zur gedruckten Kommunikation und schließlich die Digitalisierung der wis-

Dr. C. Lüthje $(\bowtie)$

Klinik für Psychiatrie und Psychotherapie, Universitätsmedizin Greifswald, Ellernholzstraße 1-2, 17475 Greifswald, Deutschland

E-Mail: corinna.luethje@uni-greifswald.de 
senschaftlichen Kommunikation. Weitere Themen sind die Wissenschaft als Massenphänomen, Zeitschriftenkrise und Open-Access-Bewegung, die Transformation des Publikationssystems sowie Open Science und die Zukunft der Wissenschaftskommunikation. Ball kommt zu dem Schluss, dass mit der Digitalisierung die Mündlichkeit wieder einen Platz findet in der wissenschaftlichen Auseinandersetzung: „Die digitalen Medien erlauben in einer dialektischen Aufhebung der früheren Formen ein kongeniales Neben- und Durcheinander von mündlichen und schriftlichen Elementen in der Diskussion und Verbreitung wissenschaftlicher Inhalte. [...] Damit gelingt dank technologischem Fortschritt erstmals die Verschmelzung mündlicher und schriftlicher Kommunikation und bietet Optionen für die Auflösung der bisherigen ,Frontstellung 'von mündlicher und schriftlicher Kommunikation.“ (S. 23).

Der Weg von mündlicher Aushandlung und Tradierung über schriftliche Fixierung bis zu ihrem Neben- und Miteinander wird in eine spannende Erzählung über die Geschichte der Wissenschaft verpackt. Leider ignoriert Ball sowohl den kommunikationswissenschaftlichen als auch den wissenschaftssoziologischen Forschungsstand. Die Rezeption der Publikationen aus den Nachbardisziplinen hätten ihm durchaus Impulse geben können. So bleibt es bei einer Deskription des Wandels der formalen Wissenschaftskommunikation, die leider nur die Oberfläche dieses Prozesses ankratzt - dies jedoch in einer durchaus ansprechenden und unterhaltsamen Form.

Funding Open Access funding enabled and organized by Projekt DEAL.

Open Access Dieser Artikel wird unter der Creative Commons Namensnennung 4.0 International Lizenz veröffentlicht, welche die Nutzung, Vervielfältigung, Bearbeitung, Verbreitung und Wiedergabe in jeglichem Medium und Format erlaubt, sofern Sie den/die ursprünglichen Autor(en) und die Quelle ordnungsgemäß nennen, einen Link zur Creative Commons Lizenz beifügen und angeben, ob Änderungen vorgenommen wurden.

Die in diesem Artikel enthaltenen Bilder und sonstiges Drittmaterial unterliegen ebenfalls der genannten Creative Commons Lizenz, sofern sich aus der Abbildungslegende nichts anderes ergibt. Sofern das betreffende Material nicht unter der genannten Creative Commons Lizenz steht und die betreffende Handlung nicht nach gesetzlichen Vorschriften erlaubt ist, ist für die oben aufgeführten Weiterverwendungen des Materials die Einwilligung des jeweiligen Rechteinhabers einzuholen.

Weitere Details zur Lizenz entnehmen Sie bitte der Lizenzinformation auf http://creativecommons.org/ licenses/by/4.0/deed.de.

Dr. Corinna Lüthje ist wissenschaftliche Mitarbeiterin an der Klinik für Psychiatrie und Psychotherapie der Universitätsmedizin Greifswald. 